\title{
Route to Chaos and Bistability Analysis of Quasi-Periodically Excited Three-Leg Supporter with Shape Memory Alloy
}

\author{
Manisekaran Varadharajan, ${ }^{1}$ Prakash Duraisamy $\left(\mathbb{D},{ }^{2}\right.$ and Anitha Karthikeyan ${ }^{3}$ \\ ${ }^{1}$ Department of Metallurgical and Materials Engineering, Defence University, Bishoftu, Ethiopia \\ ${ }^{2}$ Department of Mechanical and Industrial Engineering, EiTM, Mekelle University, Mek'ele, Ethiopia \\ ${ }^{3}$ Nonlinear Systems and Applications, Faculty of Electrical and Electronics Engineering, Ton Duc Thang University, \\ Ho Chi Minh City, Vietnam \\ Correspondence should be addressed to Prakash Duraisamy; duraiprakash83@gmail.com
}

Received 27 June 2020; Revised 2 August 2020; Accepted 8 September 2020; Published 19 September 2020

Academic Editor: Yongjian Liu

Copyright (c) 2020 Manisekaran Varadharajan et al. This is an open access article distributed under the Creative Commons Attribution License, which permits unrestricted use, distribution, and reproduction in any medium, provided the original work is properly cited.

\begin{abstract}
In this paper, the effect of quasi-periodic excitation on a three-leg supporter configured with shape memory alloy is investigated. We derived the equation of motion for the system using the supporter configuration and polynomial constitutive model of the shape memory alloys (SMAs) based on Falk model. Two sets of parameters and symmetric initial conditions are used to analyze the system. The system responded with a chaotic attractor and a strange nonchaotic attractor. Coexistence of these attractors is studied and discussed with corresponding phase portrait, bifurcation plot, and cross section of basin of attraction. We confirm the quasi-periodic excitation results with generation of strange nonchaotic attractors as discussed in the literature. The special properties like symmetricity and bistability are revealed and the parameter ranges of existence of such behaviors are discussed. The system is analyzed for different phases and the existence of bistability in martensite phase and transition phase is explained. While the system enters into austenite phase, the bistability behavior vanishes. The results provide insight knowledge into dynamical response of a quasi-periodically excited SMA leg support system and will be useful for design improvements and controller design.
\end{abstract}

\section{Introduction}

For the last two decades, shape memory alloys (SMAs) gained attention of the researchers because of their salient behavior and their versatile application in a wide range of fields [1-10]. SMAs are metallic alloys that hold the ability to regain their original shape from plastic deformation through thermal processing. SMAs holding hysteretic loop with super elasticity means the ability to recover from large elastic strains (up to $8 \%$ ) also permits the material to resist large cyclic deformations, without residual strains $[4,5]$. The mechanism behind SMAs behavior is related to martensitic phase transformation that the alloy undergoes when subjected to stress and/or temperature changes. Essentially, there are two basic crystalline states in SMAs: austenite and martensite. Martensitic transformation is a nondiffusive process, which happens due to differences between involved constituents [2]. Hence, while we design a mechanical system using SMAs, it is mandatory to investigate the behavior of the system in both martensitic phase and austenitic phase, as well as in transition phase $[6,7,9]$.

The three-leg supporter is a mechanical system that is widely used in aircraft industries and machining industries. It can provide adequate inclinations with required motion for precise movement of platform. While SMAs are introduced in three-leg platform, precise movement and maintaining the position can be easily achieved but the challenges are enormous.

Major challenge can be identified as constitutive model for representing the deformation behavior of SMAs. In order to explore all potentialities of SMAs, there is an increasing interest in the development of mathematical models capable of describing the main behaviors of these alloys. SMA thermomechanical behavior can be modeled by either 
microscopic or macroscopic points of view. Using Devonshire's theory, the unique properties of SMAs were modeled by Falk [8] and a simplified, refined one-dimensional model is formulated and discussed in [9]. This model is analyzed based on polynomial-free energy potential [10].

In 1984, Grebogi [11] found strange attractors that are not chaotic and named them Strange Nonchaotic Attractors (SNAs). The dynamics of SNAs that are neither regular nor chaotic indicate that the shape is neither a finite set of points nor piecewise differentiable manifold. The interesting phenomenon is that while the system undergoes quasi-periodically excited forces, these SNAs are generated. Many researches were carried out on such SNAs [12-21]. In [22], quasi-periodically excited nonautonomous system is investigated and various shapes of SNAs are discussed. In [23], the authors developed some no-autonomous systems with quasi-periodic coefficients and revealed more complicated dynamics of unusual types.

The three-leg supporter system does not need to be subjected to periodic excitation; hence, investigation for quasi-periodic excitation provides exact scenario of realtime situations. Coexistence of two or more attractors is not good for a mechanical system; it intricates the design and control of the system. Observing how many coexisting attractors are present in the system and investigating the parameter range and corresponding initial conditions are very important. Many literatures showed the coexistence of attractors in mechanical systems and studied their behaviors [24-27].

Motivated by the aforementioned discussion, in this paper, we analyzed the three-leg supporter with shape memory alloy for quasiperiodic forcing. The quasi-periodic excitation is achieved by taking golden ratio of irrational number. The rest of this paper is organized as follows. Section 2 provides the mathematical modeling of three-leg supporter with shape memory alloy. Section 3 deals with stability analysis. Section 4 reports the symmetricity property and the bistability property and its ranges. Finally, Section 5 summarizes the conclusion.

\section{System Modeling}

In this paper, we analyze a three-leg supporter as shown in Figure 1. The setup under consideration consists of two disks (top and base) with radii $B_{1}$ and $B_{2}$, respectively. The top disk is supported by three SMA rods having length $L$ and crosssectional area $A$. These three legs are located $120^{\circ}$ with each other on the base disk.

The masses of SMA rods are considerably less, so we assume that the mass of the structure is fully concentrated at the rigid mass disk. Applying force $P$ on the center of the rigid disk, and because of the symmetricity of the structure, we considered the vertical motion only. Based on the force

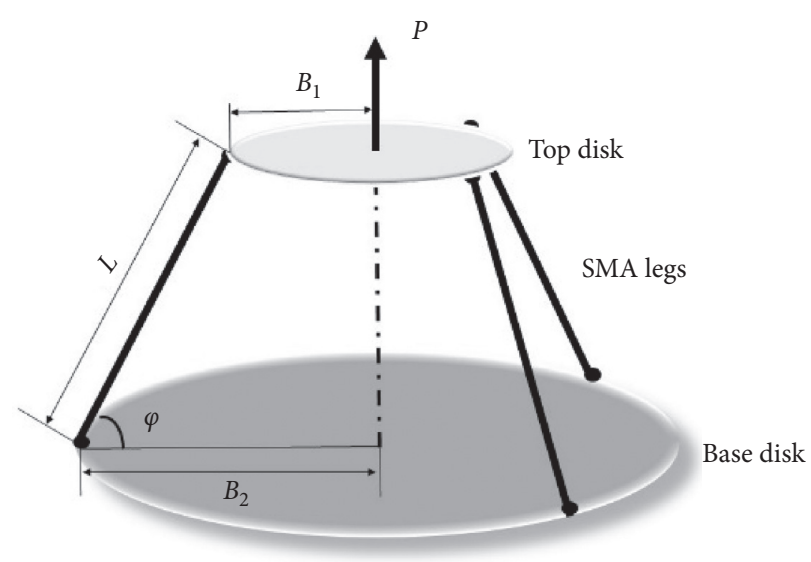

Figure 1: Three-leg supporter with shape memory alloys (SMAs).

analysis, the vertical forces can be calculated as follows: Vertical forces $=P-3$ (internal force in each bar) $\sin \varphi$.

The equation of motion is derived [28] as follows:

$$
-3 F \sin \varphi+P=m \ddot{X}+c \dot{X},
$$

where $m$ is mass and $P$ is applied force on the center of the rigid disk. $F$ is internal force in each bar, $X$ is vertical displacement, $\varphi$ is angle between the leg and the base disk, and $c$ is viscous damping coefficient.

Geometrical relations are considered in the following way:

$$
\begin{aligned}
& B=B_{2}-B_{1}, \\
& L=\sqrt{B^{2}+X^{2}} .
\end{aligned}
$$

The legs are made up of shape memory alloys (SMAs). There have been a horde of literatures that proved that SMAs possess nonlinear behavior because of their hysteretic nature while subjected to temperature changes. In order to consider such phenomenon, various constitutive models were developed. There are different ways to describe the thermomechanical behavior of SMAs. Here, we considered the polynomial constitutive model proposed by Falk [8]. He formulated the below-mentioned fifth-degree polynomial constitutive relation for analyzing the nonlinear behavior of SMAs:

$$
\sigma=a_{1}\left(T-T_{M}\right) \varepsilon-a_{2} \varepsilon^{3}+a_{3} \varepsilon^{5},
$$

where $\varepsilon=$ axial strain $=L /\left(L_{0}-1\right)=\cos \varphi_{0} /(\cos \varphi-1), T_{M}$ is the temperature below which the martensitic phase is stable, $T$ is temperature, $a_{1}, a_{2}, a_{3}$ are material constants, and $T_{A}$ is the temperature above which austenite is stable $=T_{M}+1 /\left(4\left(a_{2}^{2} / a_{1} a_{3}\right)\right)$.

By substituting geometrical relations equation (2) and fifth-degree polynomial constitutive relation (3) in equation 
(1), we can get the equation of motion of the three-leg supporter with shape memory alloy as follows:

$$
m \ddot{X}+c \dot{X}+\frac{3 A X}{L_{0}}\left\{\begin{array}{c}
{\left[a_{1}\left(T-T_{M}\right)-3 a_{2}+5 a_{3}\right]} \\
+\left[-a_{1}\left(T-T_{M}\right)+a_{2}-a_{3}\right] \frac{L_{0}}{\sqrt{X^{2}+B^{2}}}+\left[3 a_{2}-10 a_{3}\right] \frac{\sqrt{X^{2}+B^{2}}}{L_{0}} \\
+\left[-a_{2}+10 a_{3}\right] \frac{X^{2}+B^{2}}{L_{0}^{2}}-5 a^{3} \frac{\sqrt[3]{X^{2}+B^{2}}}{L_{0}^{3}}+a_{3} \frac{\left(X^{2}+B^{2}\right)^{2}}{L_{0}^{4}}
\end{array}\right\}=P(t) .
$$

$P(t)$ is the force applied to the three-leg supporter. Most of the literatures discussed the response of the system for periodic excitation but quasi-excitation is the one that will give more real situations for analyzing the nonlinear behaviors keeping this in mind. In this paper, we consider quasi-periodic excitation in the following form:

$$
P(t)=P_{0}\left[\sin \left(\Omega_{1} t\right)+\sin \left(\Omega_{2} t\right)\right] .
$$

$$
\begin{aligned}
& \dot{x}=y, \\
& \dot{y}=\gamma\left[\sin \left(\Omega_{1} \tau\right)+\sin \left(\Omega_{2} \tau\right)\right]-\eta y+x\left\{\begin{array}{c}
-\left[(\theta-1)-3 \alpha_{2}-5 \alpha_{3}\right]+\frac{\left[(\theta-1)-\alpha_{2}+\alpha_{3}\right]}{\sqrt{\left(x^{2}+b^{2}\right)}} \\
-\left[3 \alpha_{2}-10 \alpha_{3}\right] \sqrt{\left(x^{2}+b^{2}\right)}+\left[\alpha_{2}-10 \alpha_{3}\right]\left(x^{2}+b^{2}\right) \\
+5 \alpha_{3} \sqrt[3]{\left(x^{2}+b^{2}\right)}-\alpha_{3}\left(x^{2}+b^{2}\right)^{2}
\end{array}\right\},
\end{aligned}
$$

where $\omega_{0}^{2}=\left(3 A a_{1} T_{M} / m L_{0}\right), \gamma=\left(P_{0} / m L_{0} \omega_{0}^{2}\right), \eta=\left(c / m \omega_{0}\right)$, $\alpha_{2}=\left(a_{2} / a_{1} T_{M}\right), \quad \alpha_{3}=\left(a_{3} / a_{1} T_{M}\right), \quad \Omega_{1}=\left(\omega_{1} / \omega_{0}\right)$, $\Omega_{2}=\left(\omega_{2} / \omega_{0}\right), b=\left(B / L_{0}\right), \theta_{A}=\left(T_{A} / T_{M}\right), x=\left(X / L_{0}\right), \theta=$ $\left(T / T_{M}\right)$, and $\tau=\omega_{0} t$.
The necessary condition to get quasi-excitation, $\left(\Omega_{1} / \Omega_{2}\right)$ should be irrational number [20,29]. For our analysis, we take the golden ratio of irrational number $\Omega_{1}=0.5$, $\Omega_{2}=((\sqrt{5}-1) / 2)$.

The dimensionless equation is derived from the equation of motion:

\section{Stability Analysis}

In order to find the equilibrium point, we considered an unforced system from equation (6). We discarded the forcing term $\gamma\left[\sin \left(\Omega_{1} \tau\right)+\sin \left(\Omega_{2} \tau\right)\right]$. Then the equations are equated to zero. The equations become as follows:

$$
\begin{aligned}
& 0=y, \\
& 0=-\eta y+x\left\{\begin{array}{c}
-\left[(\theta-1)-3 \alpha_{2}-5 \alpha_{3}\right]+\frac{\left[(\theta-1)-\alpha_{2}+\alpha_{3}\right]}{\sqrt{\left(x^{2}+b^{2}\right)}} \\
-\left[3 \alpha_{2}-10 \alpha_{3}\right] \sqrt{\left(x^{2}+b^{2}\right)}+\left[\alpha_{2}-10 \alpha_{3}\right]\left(x^{2}+b^{2}\right) \\
+5 \alpha_{3} \sqrt[3]{\left(x^{2}+b^{2}\right)}-\alpha_{3}\left(x^{2}+b^{2}\right)^{2}
\end{array}\right\} .
\end{aligned}
$$




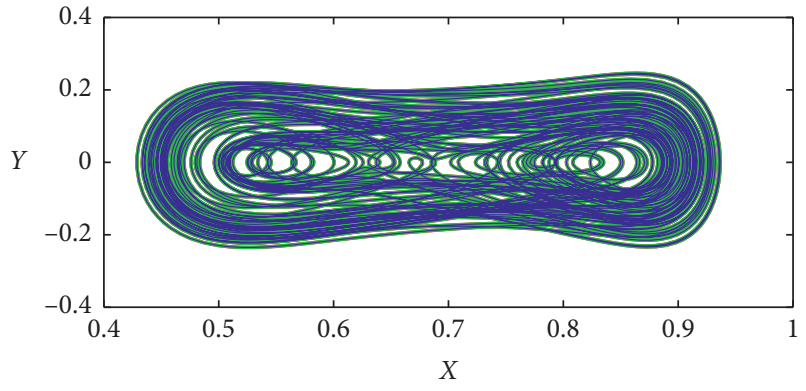

(a)

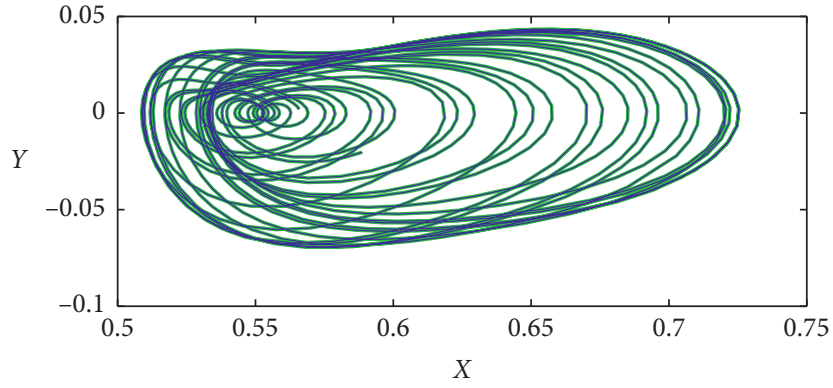

(b)

Figure 2: Phase portrait of system (7): for (a) $\eta=0.1$ and initial condition $[0.6,0]$ chaotic attractor and for (b) $\eta=1.5$ and initial condition $[0.5,2]$ attracting tori.

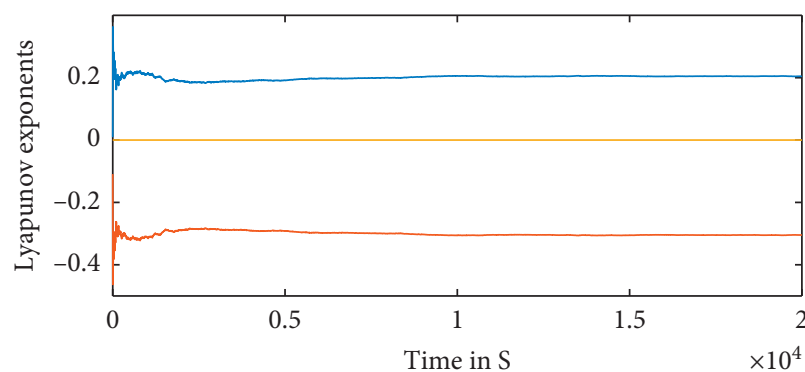

(a)

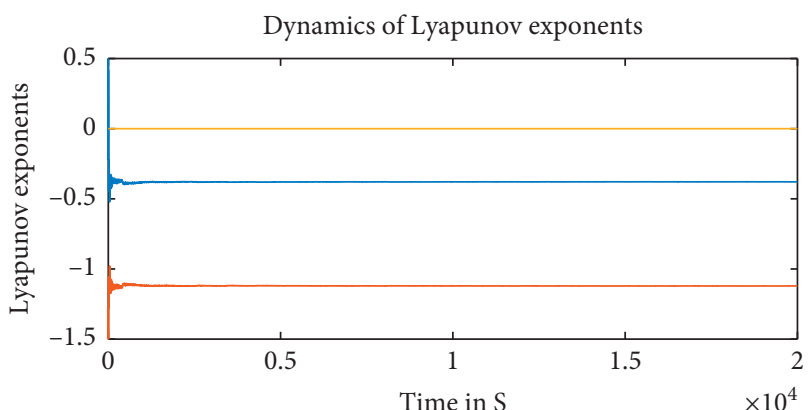

(b)

FIGURE 3: Lyapunov exponents. (a) For $\eta=0.1$ and initial condition of $[0.6,0]$; (b) for $\eta=1.5$ and initial condition of [0.5, 2].

For the parameter values,

$\alpha_{2}=124 ; \alpha_{3}=14505 ; \gamma=0.045 ; b=0.7071 ; \theta=0.69 ; \eta=0.1$,

$$
J=\left[\begin{array}{cc}
0 & 1 \\
A_{1} & -\eta
\end{array}\right],
$$

where

and equilibrium point $\{-0.00113,0\}$ is found.

We derived the Jacobian matrix for equation (7) given as

$$
A_{1}=\left\{\begin{array}{l}
3 a_{2}-5 a_{3}-\theta-\frac{a_{2}-a_{3}-\theta+1}{\sqrt{b^{2}+x^{2}}}-a_{3}\left(b^{2}+x^{2}\right)^{2}+5 a_{3} \sqrt[3]{b^{2}+x^{2}}-\left(3 a_{2}-10 a_{3}\right) \sqrt{b^{2}+x^{2}} \\
+\left(a_{2}-10 a_{3}\right)\left(b^{2}+x^{2}\right)+\frac{x\left(2 x\left(a_{2}-10 a_{3}\right)\right)-\left(x\left(3 a_{2}-10 a_{3}\right)\right)}{\sqrt{b^{2}+x^{2}}}-4 a_{3} x\left(b^{2}+x^{2}\right) \\
+\frac{x\left(a_{2}-a_{3}-\theta+1\right)}{\sqrt[3]{b^{2}+x^{2}}}+15 a_{3} x \sqrt{b^{2}+x^{2}}+1 .
\end{array}\right.
$$

The characteristic equation is formulated for the Jacobian matrix on the equilibrium point:

$$
\lambda^{2}+0.1 \lambda-39.6866=0 .
$$

Corresponding eigenvalues are found as

$$
\lambda_{1}=6.2499 ; \lambda_{2}=-6.3499 \text {, }
$$

and we have eigenvalues with one positive and negative value; hence, the equilibrium point can be a saddle node.

\section{Dynamic Analysis}

In this paper, we analyzed system (6) for different $\theta$ values to study the behavior during martensitic phase $(\theta=0.69)$, transition phase $(\theta=1.04)$, and austenitic phase $(\theta=1.30)$. 


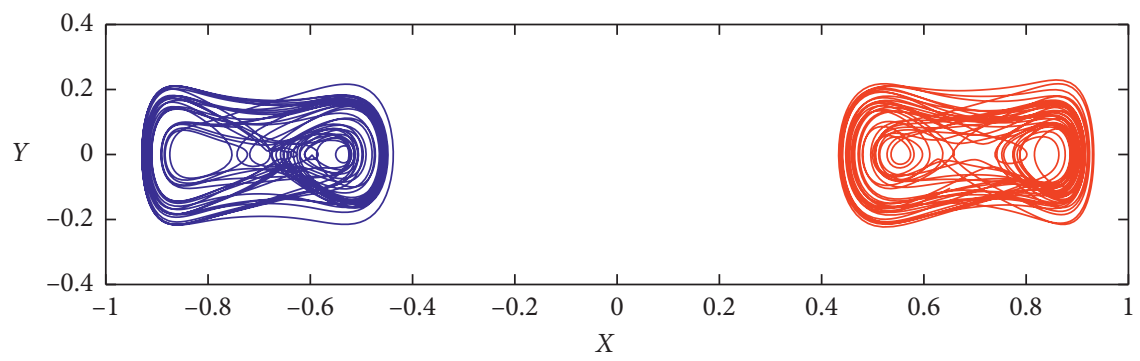

Figure 4: Chaotic attractors for initial conditions of $[0.6,0]$ and $[-0.5,0]$.

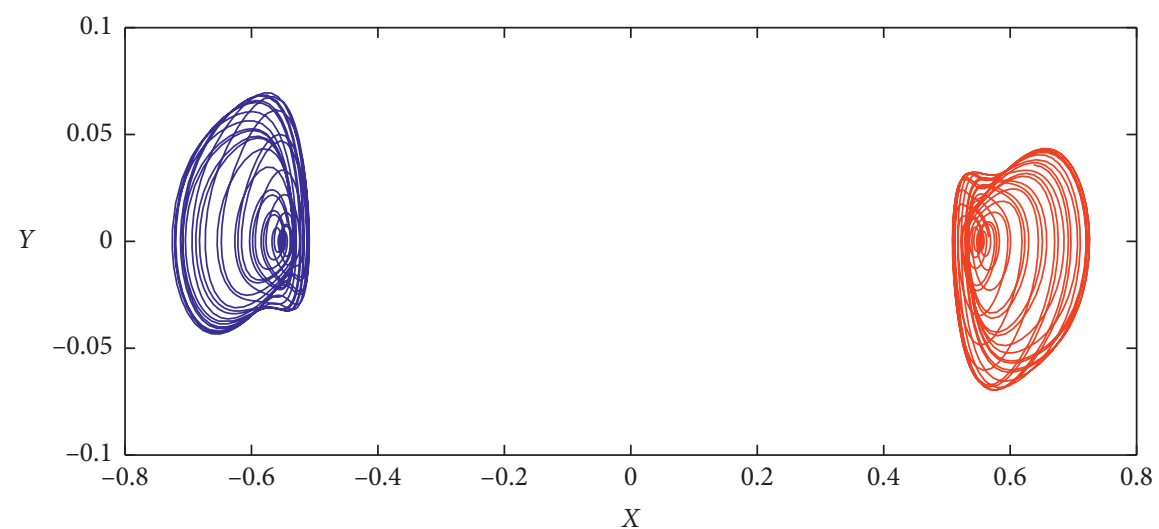

FIGURE 5: Tori attractors for initial conditions of $[0.5,2]$ and $[-0.5,-2]$.
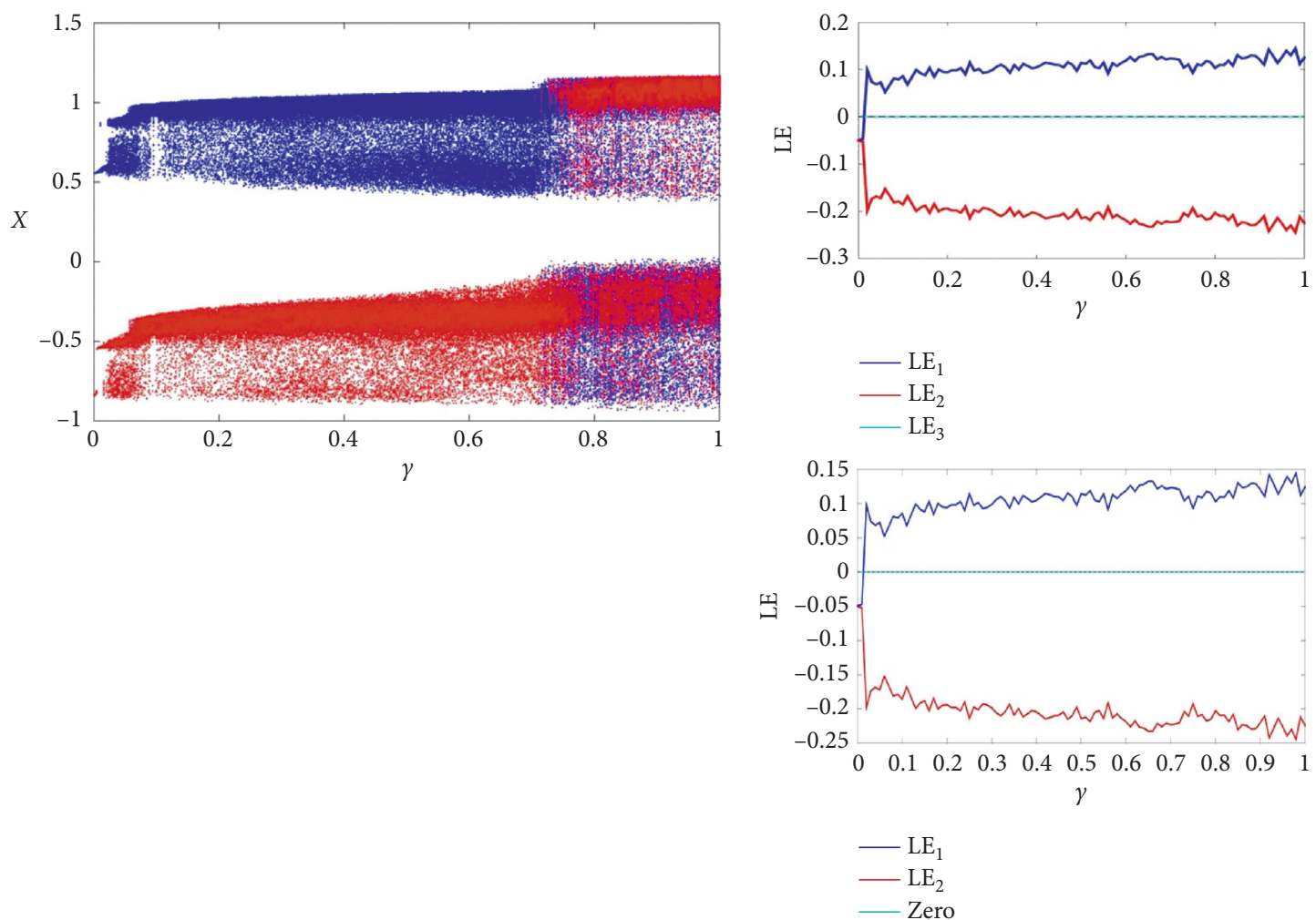

(a)

(b)

Figure 6: (a) Bistability of system (7) with the blue plots showing the forward continuation (increase parameter $\gamma$ from 0 to 1 ) and the red plots showing backward continuation (decrease parameter $\gamma$ from 1 to 0 ) with reinitialization of the initial conditions. (b) Corresponding Lyapunov exponents. 

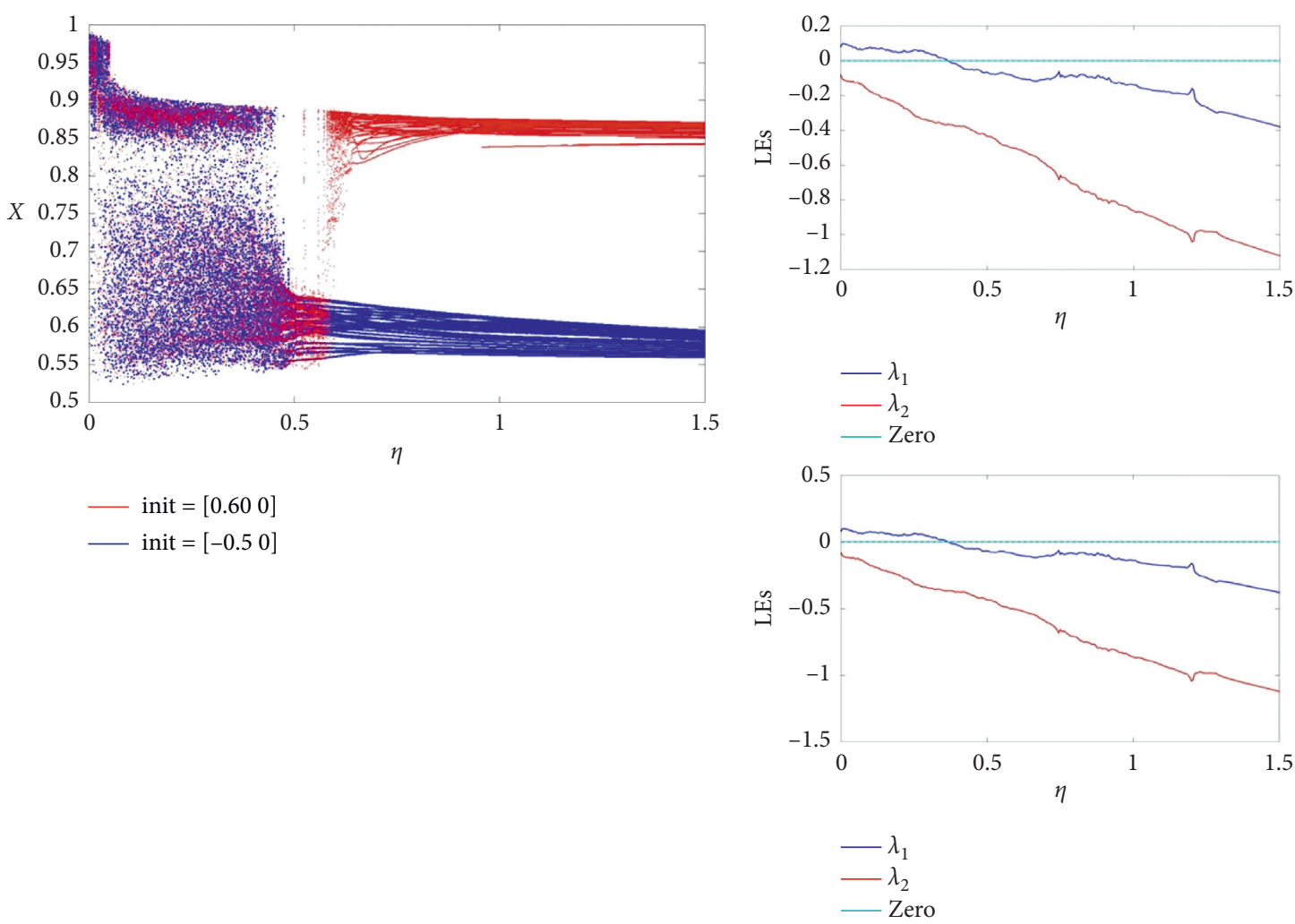

(a)

(b)

Figure 7: (a) Bistability of system (8) with the blue plots showing the forward continuation (increase parameter $\eta$ from 0 to 1.5 ) and the red plots showing backward continuation (Decrease parameter from 1 to 0 ) with reinitialization of the initial conditions. (b) Corresponding Lyapunov exponents.

4.1. Case (A) during Martensitic Phase. In this case, we considered low temperature $(\theta=0.69)$, and dynamical behavior of the system during martensitic phase is analyzed. In the model, we supplied $a_{2}=124, a_{3}=14505, b=0.7071$, $\gamma=0.045, \Omega_{1}=0.5$, and $\Omega_{2}=((\sqrt{5}-1) / 2)$. Meanwhile, $\eta=0.1$ and, for the initial condition $[0.6,0]$, chaotic attractor is observed. Also, we have $\eta=1.5$ and, for the initial condition $[0.5,2]$, attracting tori is found. We present the phase portraits of chaotic attractor and tori in Figures 2(a) and 2(b) correspondingly.

The Lyapunov exponents are estimated using Wolf's algorithm [30], for the runtime of 20,000 s. Figure 3(a) shows a positive Lyapunov exponent with value of 0.204674 , and the positive Lyapunov exponent confirms that it is a chaotic attractor. In Figure 3(b), both Lyapunov exponents have negative value; hence, they are concluded as attracting tori.

We extend our investigation for special properties; in Figure 4, we show that the system holds symmetric chaotic attractor for initial conditions of $[0.6,0]$ and $[-0.5,0]$, which clearly explains the sensitiveness of the system with initial conditions. Similarly, in Figure 5, we show symmetricity of the attracting tori.

System (7) is analyzed for the property of bistability. In this section, we perform the bifurcation analysis for system (7). Bistability is dangerous in mechanical systems and makes the controllability complex. Hence, it is mandatory

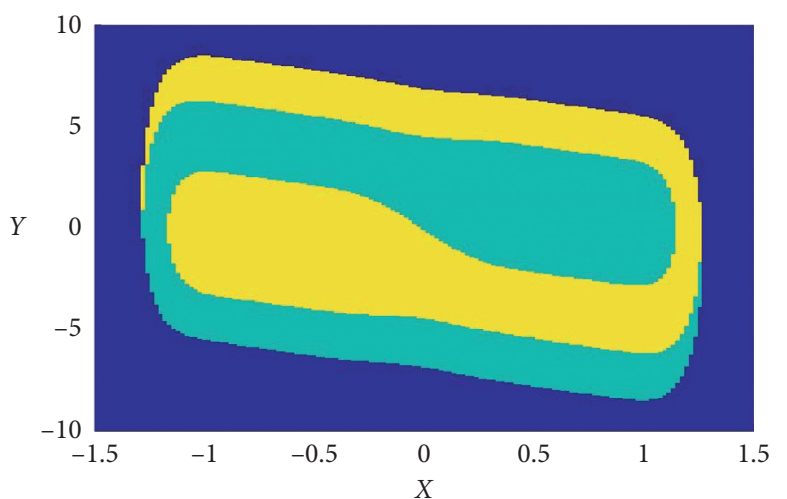

FIgURE 8: Cross section of the basins of attraction of the two coexisting attractors in the $x$-y-plane.

to analyze the existence of bistability in mechanical systems and explicate the region of such coexistence of attractors. We considered $\gamma$ as bifurcation parameter and keep all the other parameters fixed. By plotting local maxima of the coordinate $x$ in terms of the increasing (or decreasing) parameter with step size of 0.0003 , this approach is known as forward and backward continuation, representing a simple way to localize the window in which the system possesses multistability. The existence of 


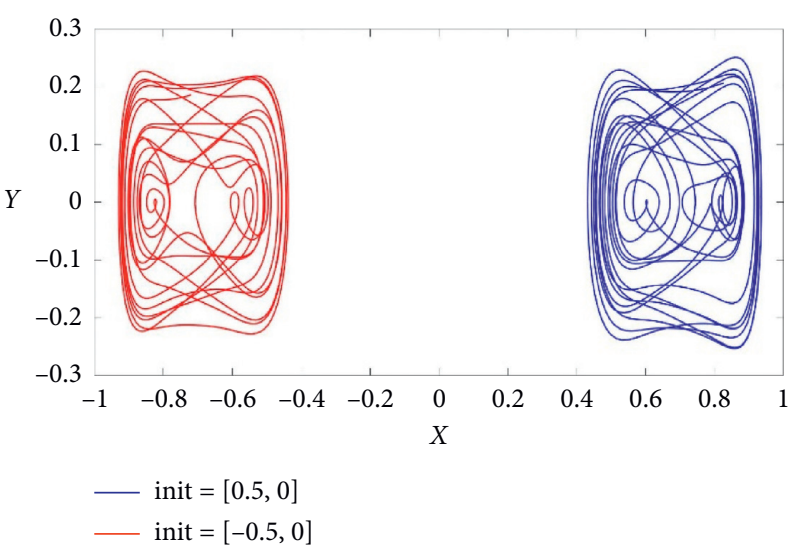

(a)

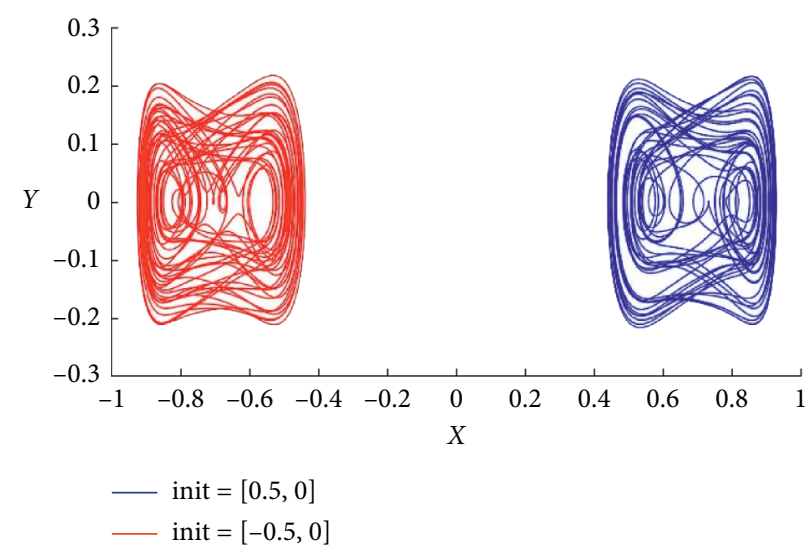

(b)

Figure 9: Phase portrait of system (7) for $\theta=1.04$ and $\gamma=0.045$. (a) $\eta=0.01$; (b) $\eta=0.1$. Remaining parameters are kept as in (9).

bistability can be confirmed by comparing the forward (Figure 6, blue) and backward (Figure 6, red) bifurcation diagrams. The corresponding Lyapunov exponents are plotted in Figure 6(b).

The bifurcation plot in Figure 7(a) portrays the existence of bistability when varying the $\eta$ parameter. The maximum Lyapunov exponents for forward continuation are plotted with blue color and backward continuation is plotted with red color. Corresponding Lyapunov exponents are plotted in Figure 7(b).

A necessary tool for analyzing the bistability (coexistence of attractors) is the basin of attraction. All attractors, whether they are periodic oscillation, attracting tori, or strange attractors, are surrounded by a basin of attraction representing the set of initial conditions in the state space whose orbits approach and map out the attractor as time approaches infinity. Figure 8 depicts the cross section of the basin of attraction of two coexisting attractors in the $x-y$-plane. The plott is obtained for the parameter values as it is in (9). Initial conditions in the blue region lead to unbounded orbits, those in the yellow region lead to the chaotic attractor, and those in the cyan region lead to the attracting tori.

4.2. Case (B) during Transition Phase. The intermediate temperature $(\theta=1.04)$ where both austenite and martensite are stable is now considered. Unlike the previous case, the parameter $\eta$ does not have a big influence in this case. We plotted the phase portraits of the system for $\eta=0.01$ (Figure 9) and $\eta=0.1$ (Figure 10), both showing similar chaotic attractor. But we could observe symmetric properties from Figures 9(a) and 9(b).

However, variation in parameter $\gamma$ still holds bistability. Figure 10 shows the existence of different oscillation in the system dynamics. We showed the bifurcation diagram for the range $0 \leq \gamma \leq 0.05$; we can clearly identify the bistability portions 0 to 0.005 from Figure 11. In Figure 12, we present the basin of attraction in order to show the different attractors.

4.3. Case (C) during Austenitic Phase. In this case, the response of the system in higher temperature $(\theta=1.30)$ is

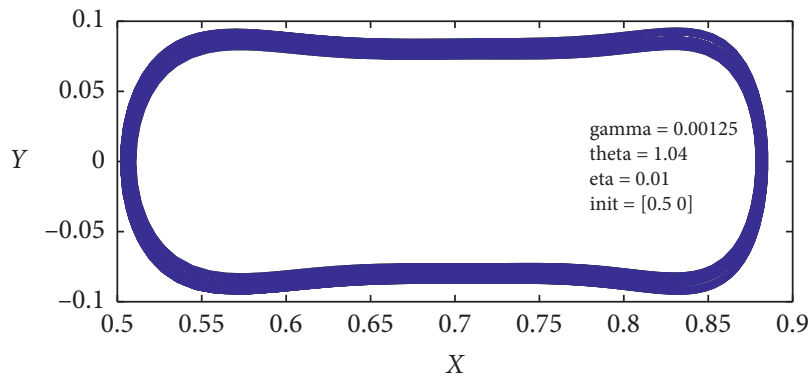

Figure 10: Phase portrait of system (7) for $\theta=1.04, \eta=0.01$, and $\gamma=0.00125$. Remaining parameters are kept as in (9).

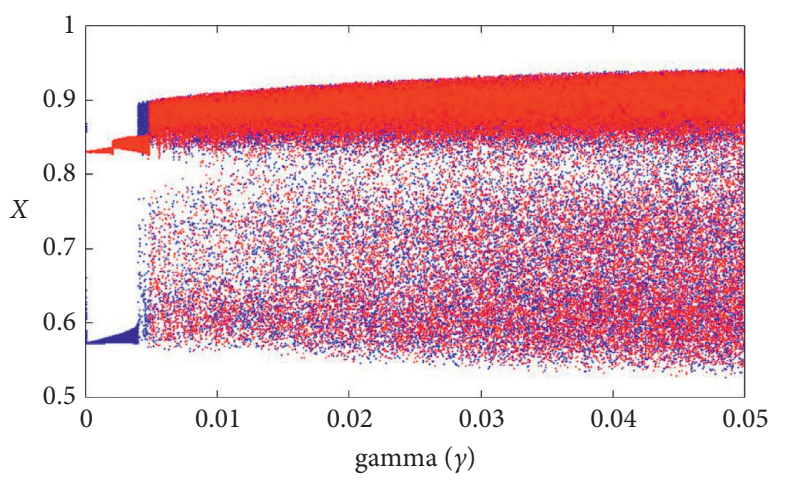

Figure 11: Bifurcation diagram for $\theta=1.04$ and $\eta=0.01$ with the blue plots showing the forward continuation (increase the parameter $0 \leq \gamma \leq 0.05)$ and the red plots showing backward continuation.

investigated, where the austenitic phase is stable. During this phase, the system shows sustainable chaotic oscillations for different $\eta$ values as shown in Figure 12. Influence of parameter $\gamma$ is analyzed using the bifurcation diagrams as shown in Figures 13 and 14. In Figure 13, we could see that $\gamma$ varied from 0 to 1 and $\eta$ is taken as 0.01 . Chaotic oscillation is observed and does not show bistability behavior. Similarly, 


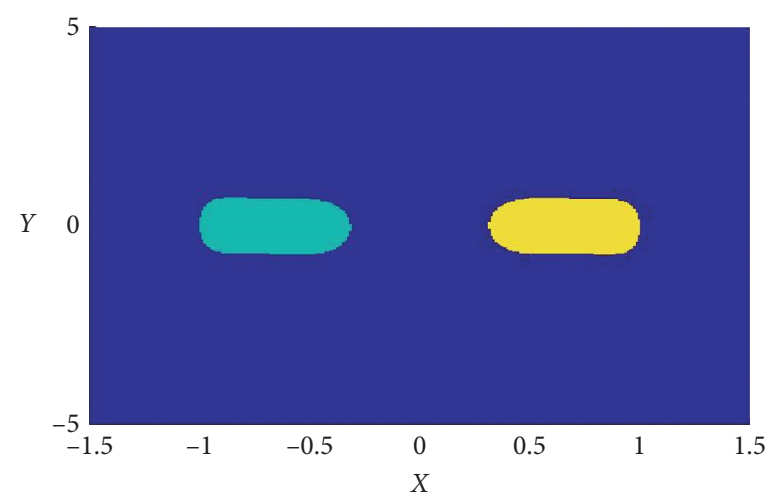

FIgURE 12: Cross section of the basins of attraction of the two coexisting attractors in the $x$-y-plane (Case B).

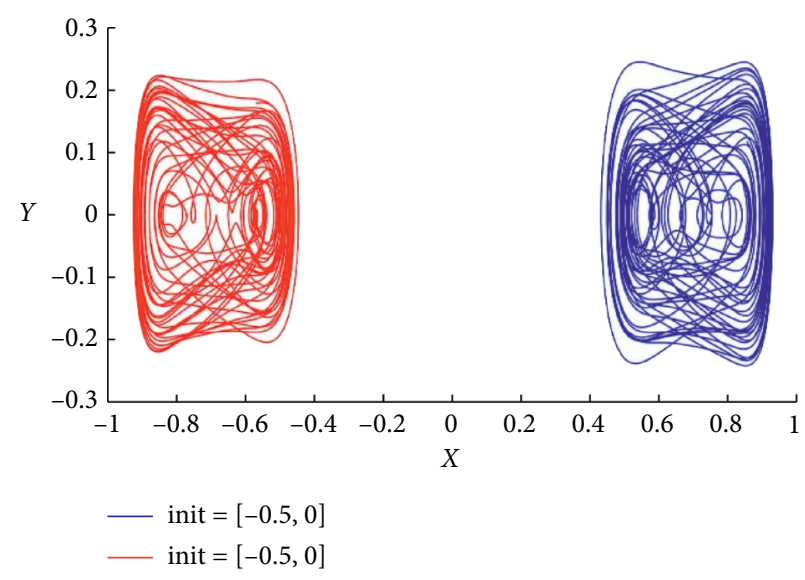

(a)

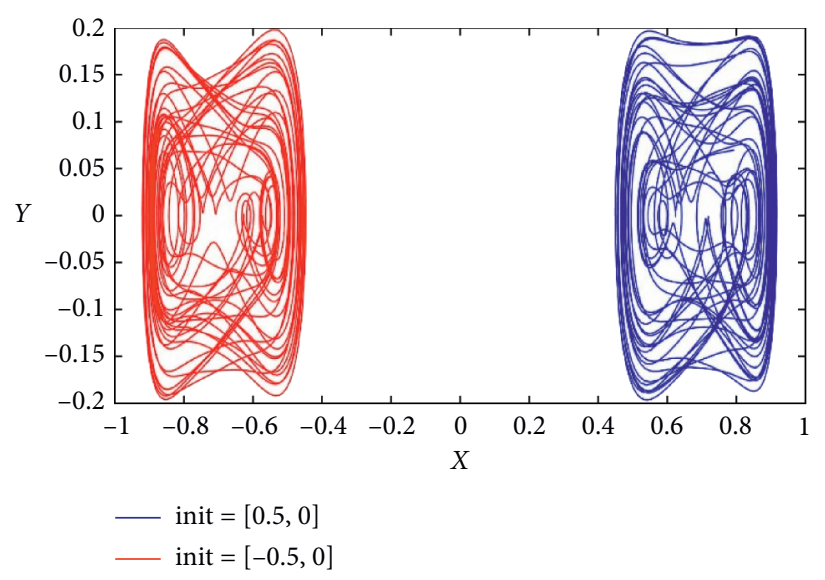

(b)

FIGURE 13: Phase portrait of system (7) for $\theta=1.30$ and $\gamma=0.045$. (a) $\eta=0.01$; (b) $\eta=0.1$. Remaining parameters are kept as in (9).

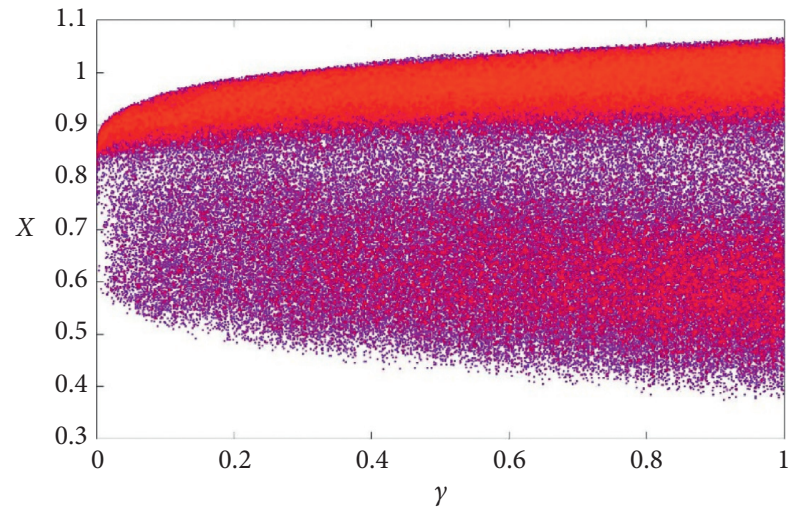

Figure 14: Bifurcation diagram of system (7) for $\theta=1.30$ and $\eta=$ 0.01 with the blue plots showing the forward continuation (increase the parameter $0 \leq \gamma \leq 1)$ and the red plots showing backward continuation.

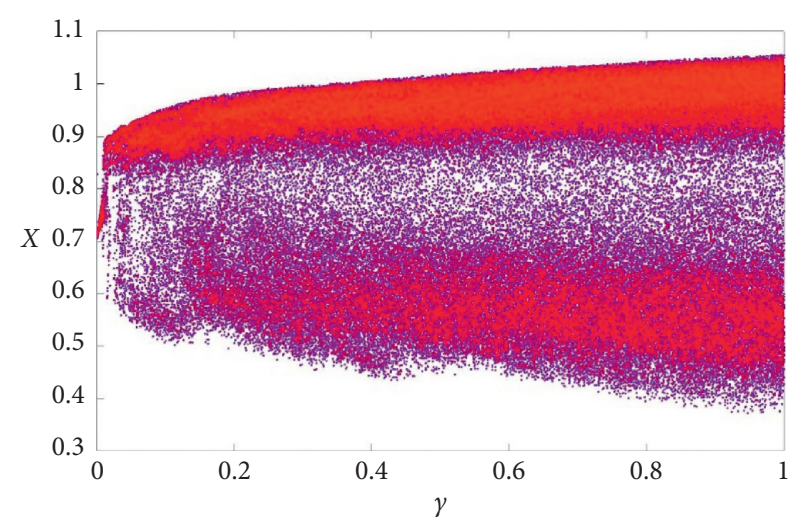

FIGURE 15: Bifurcation diagram of system (7) for $\theta=1.30$ and $\eta=$ 0.1 with the blue plots showing the forward continuation (increase the parameter $0 \leq \gamma \leq 1$ ) and the red plots showing backward continuation. 


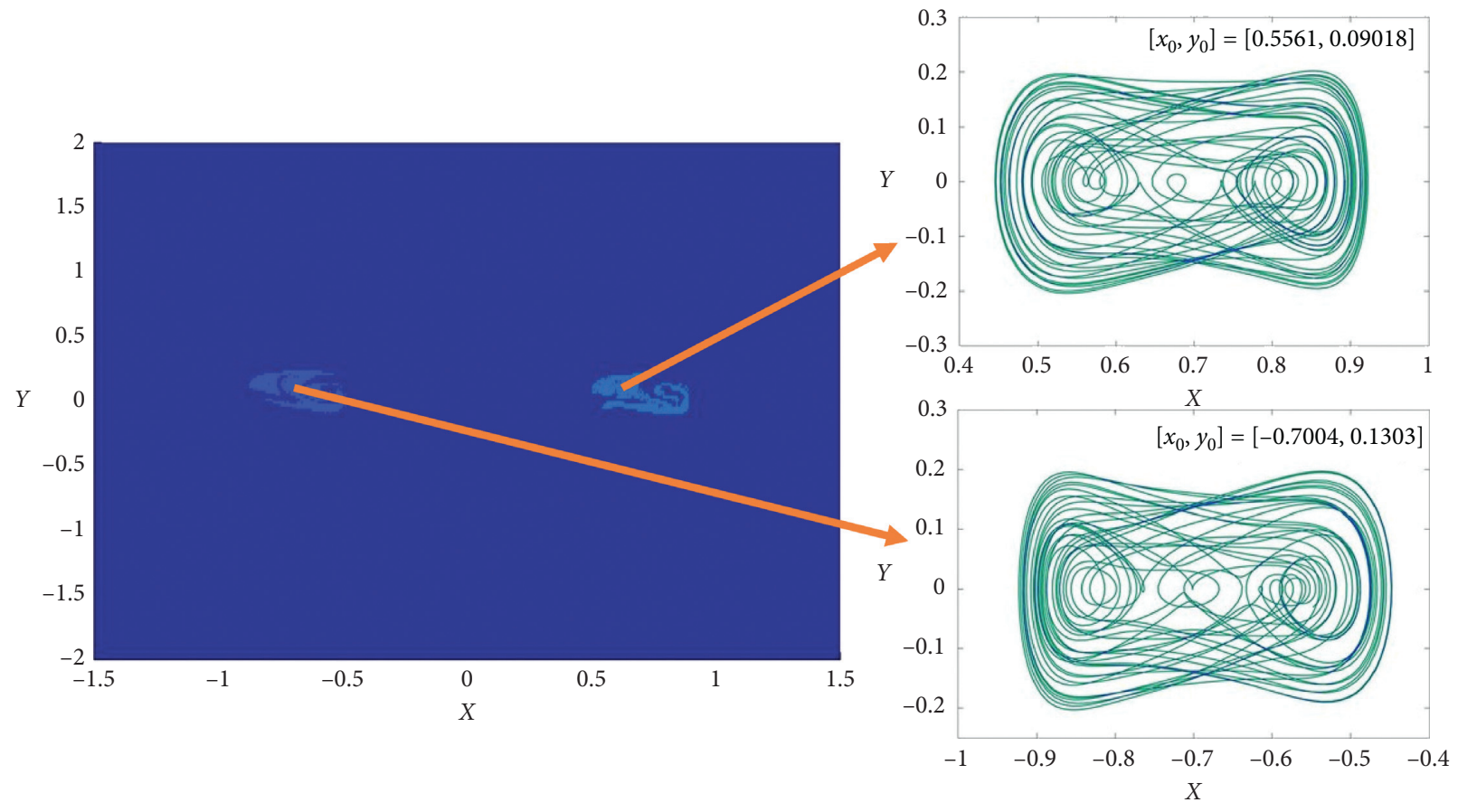

(a)

(b)

FiguRE 16: Cross section of the basins of attraction of the two coexisting attractors in the $x$ - $y$-plane (Case C).

while we increase the $\eta$ value to 0.1 , the system does not show any bistability. Basin of attraction is also provided in Figure 15. It is worth noting that the three-leg supporter system suffers from bistability during martensitic (Figure 16) and transition phases but does not suffer from it if it operates in austenitic phase (higher temperature).

\section{Conclusion}

In this paper, a quasi-periodically excited three-leg supporter with shape memory alloy is investigated for bistability behavior. The existence of chaotic strange attractor and an attracting tori has been evolved and depicted in phase portraits; hence, it is clear that considering quasiperiodic excitation holds the property of bistability. Chaotic strange attractor is confirmed by showing positive Lyapunov exponents. The presence of symmetric behavior is revealed and shown for the corresponding initial conditions; this kind of behavior is interesting for controlling the system. Bifurcation plots presented in Section 4 show the behavioral changes of $\gamma$ and $\eta$; hence, we can easily understand the bistability nature of the system for particular range of values. Cross section of basin of attraction is plotted for showing the pool of initial conditions, where we can get the chaotic attractor and the attracting tori. More importantly, in our study, we showed that the bistability behavior is present only during martensitic and transition phases and not in austenitic phase. We firmly believe that the analysis will be very helpful during designing and developing control algorithm of three-leg platform with shape memory alloys.

\section{Data Availability}

The data used to support the findings of this paper are included within the manuscript.

\section{Conflicts of Interest}

The authors declare that there are no conflicts of interest regarding the publication of this paper.

\section{References}

[1] A. S. Marcelo, "Nonlinear dynamics and chaos in shape memory alloy systems," International Journal of Non-linear Mechanics, vol. 70, pp. 2-19, 2015.

[2] R. J. Wasilevski, "On the nature of the martensitic transformation," Metallurgical Transactions A, vol. 6, pp. 14051418, 1975.

[3] K. Otsuka and X. Ren, "Recent developments in the research of shape memory alloys," in Institute of Materials Science, University of Tsukuba, Tsukuba, Japan, 1999.

[4] C. E. Borroni-Bird, "Smarter vehicles. smart structures and materials 1997: industrial and commercial applications of smart structures technologies," in Proceedings of the SPIE-the International Society for Optical Engineering, San Diego, CA, USA, March 1997.

[5] T. Borden, "Shape memory alloys: forming a tight fit," Mechanical Engineering, vol. 113, no. 10, pp. 66-72, 1991.

[6] M. A. Savi and A. M. B. Braga, "Chaotic vibrations of na oscillator with shape memory," Journal of the Brazilian Society of Mechanical Sciences and Engineering, vol. 15, no. 1, pp. 1-20, 1993.

[7] M. A. Savi and A. M. B. Braga, "Chaotic response of a shape memory oscillator with internal constraints," in Proceedings of 
the XII the Brazilian Congress of Mechanical Engineering (COBEM 93-ABCM), pp. 33-36, Brasilia, Brazil, December 1993.

[8] F. Falk, "Model free-energy, mechanics and thermodynamics of shape memory alloys," ACTA-Metallurgica, vol. 28, no. 12, pp. 1773-1780, 1980.

[9] F. Falk and P. Konopka, "Three-dimensional Landau theory describing the martensitic transformation of shape memory alloys," Journal De Physique, vol. 2, pp. 61-77, 1990.

[10] P. Alberto and A. savi Marcelo, "An overview of constitutive models for shape memory alloys," Mathematical Problems in Engineering, vol. 2006, no. 43, Article ID 056876, 2006.

[11] C. Grebogi, E. Ott, S. Pelikan, and J. A. Yorke, "Strange attractors that are not chaotic," Physica D: Nonlinear Phenomena, vol. 13, no. 1-2, pp. 261-268, 1984.

[12] Z. I. Bezhaeva and V. I. Oseledets, "An example of a strange nonchaotic attractor," Functional Analysis and Its Applications, vol. 30, no. 4, pp. 223-229, 1996.

[13] M. Ding, C. Grebogi, and E. Ott, "Evolution of attractors in quasiperiodically forced systems: from quasiperiodic to strange nonchaotic to chaotic," Physical Review A, vol. 39, no. 5, pp. 2593-2598, 1989.

[14] U. Feudel, J. Kurths, and A. S. Pikovsky, "Strange non-chaotic attractor in a quasiperiodically forced circle map," Physica D: Nonlinear Phenomena, vol. 88, no. 3-4, pp. 176-186, 1995.

[15] J. F. Heagy and S. M. Hammel, "The birth of strange nonchaotic attractors," Physica D: Nonlinear Phenomena, vol. 70, no. 1-2, pp. 140-153, 1994.

[16] À. Jorba, J. C. Tatjer, C. Núñez, and R. Obaya, "Old and new results on strange nonchaotic attractors," International Journal of Bifurcation and Chaos, vol. 17, no. 11, pp. 38953928, 2007.

[17] G. Keller, "A note on strange nonchaotic attractors," Fundamenta Mathematicae, vol. 151, no. 2, pp. 139-148, 1996.

[18] S. P. Kuznetsov, A. S. Pikovsky, and U. Feudel, "Birth of a strange nonchaotic attractor: a renormalization group analysis," Physical Review E, vol. 51, pp. 1629-1632, 1995.

[19] F. J. Romeiras, A. Bondeson, E. Ott, T. M. Antonsen, and C. Grebogi, "Quasiperiodically forced dynamical systems with strange nonchaotic attractors," Physica D: Nonlinear Phenomena, vol. 26, no. 1-3, pp. 277-294, 1987.

[20] F. J. Romeiras, A. Bondeson, E. Ott, T. M. Antonsen, and C. Grebogi, "Quasiperiodic forcing and the observability of strange nonchaotic attractors," Physica Scripta, vol. 40, no. 3, pp. 442-444, 1989.

[21] K. V. Avramov and O. V. Gendelman, "Quasiperiodic forced vibrations of a beam interacting with a nonlinear spring," Acta Mechanica, vol. 19, no. 2, pp. 17-35, 2007.

[22] Xu Zhang, "Dynamics of nonautonomous ordinary differential equations with quasi-periodic coefficients," International Journal of Bifurcation and Chaos, vol. 27, no. 6, 2017.

[23] X. Zhang and G. Chen, "Chaotic and non-chaotic strange attractors of a class of non-autonomous systems," Chaos: An Interdisciplinary Journal of Nonlinear Science, vol. 28, no. 2, Article ID 023102, 2018.

[24] U. Feudel, C. Grebogi, L. Poon, and J. A. Yorke, "Dynamical properties of a simple mechanical system with a large number of coexisting periodic attractors," Chaos, Solitons \& Fractals, vol. 9, no. 1-2, pp. 171-180, 1998.

[25] K. Rajagopal, A. Karthikeyan, and P. Duraisamy, "Bifurcation analysis and chaos control of a fractional order portal frame with nonideal loading using adaptive sliding mode control," Shock and Vibration, vol. 2017, Article ID 2321060, 2017.
[26] K. Rajagopal, D. Prakash, R. Weldegiorgis, and A. Karthikeyan, "Multistability in horizontal platform system with and without time delays," Shock and Vibration, vol. 2018, Article ID 1092812, 2018.

[27] P. Prakash, K. Rajagopal, J. P. Singh, and B. K. Roy, "Megastability in a quasi-periodically forced system exhibiting multistability, quasi-periodic behaviour, and its analogue circuit simulation," AEU-International Journal of Electronics and Communications, vol. 92, pp. 111-115, 2018.

[28] Z. Huang, C. Du, and Y. Li, "Bifurcation and chaos of a shape memory alloy supporter," in Proceedings of the 2008 7th World Congress on Intelligent Control and Automation, Chongqing, China, June 2008.

[29] M. Agrawal, A. Prasad, and R. Ramaswamy, "Quasiperiodic forcing of coupled chaotic systems," Physical Review E, vol. 81, Article ID 026202, 2010.

[30] A. Wolf, J. B. Swift, H. L. Swinney, and J. A. Vastano, "Determining Lyapunov exponents from a time series," Physica D: Nonlinear Phenomena, vol. 16, no. 3, pp. 285-317, 1985. 